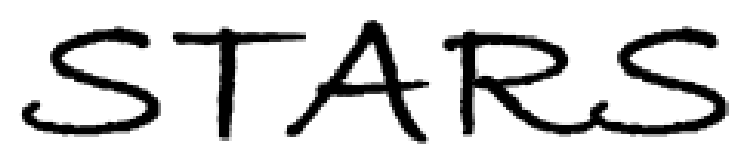

University of Central Florida

STARS

$1-1-1998$

\title{
All-optical mode mixer spatial switch based on cascading in lithium niobate
}

\author{
Yongsoon Baek \\ University of Central Florida \\ Roland Schiek \\ University of Central Florida \\ George Stegeman \\ University of Central Florida \\ Gaetano Assanto \\ W. Sohler
}

Find similar works at: https://stars.library.ucf.edu/facultybib1990 University of Central Florida Libraries http://library.ucf.edu

This Article is brought to you for free and open access by the Faculty Bibliography at STARS. It has been accepted for inclusion in Faculty Bibliography 1990s by an authorized administrator of STARS. For more information, please contactSTARS@ucf.edu.

\section{Recommended Citation}

Baek, Yongsoon; Schiek, Roland; Stegeman, George; Assanto, Gaetano; and Sohler, W., "All-optical mode mixer spatial switch based on cascading in lithium niobate" (1998). Faculty Bibliography 1990s. 2171. https://stars.library.ucf.edu/facultybib1990/2171

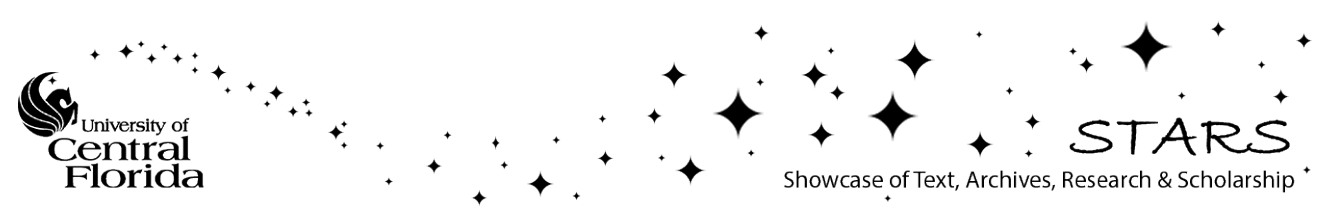




\section{All-optical mode mixer spatial switch based on cascading in lithium niobate}

Cite as: Appl. Phys. Lett. 72, 3405 (1998); https://doi.org/10.1063/1.121647

Submitted: 19 March 1998 . Accepted: 27 April 1998 . Published Online: 23 June 1998

Yongsoon Baek, Roland Schiek, George Stegeman, Gaetano Assanto, and W. Sohler

\section{ARTICLES YOU MAY BE INTERESTED IN}

Optical waveguides in lithium niobate: Recent developments and applications

Applied Physics Reviews 2, 040603 (2015); https://doi.org/10.1063/1.4931601

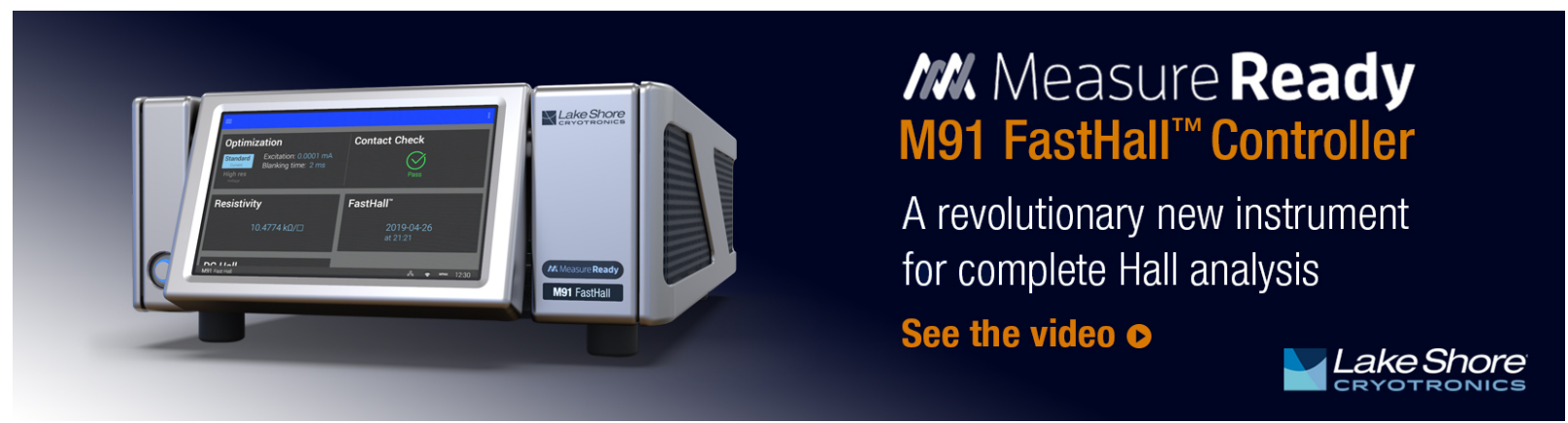




\title{
All-optical mode mixer spatial switch based on cascading in lithium niobate
}

\author{
Yongsoon Baek, ${ }^{\text {a) }}$ Roland Schiek, and George Stegeman \\ CREOL, University of Central Florida, Orlando, Florida 32816-2700 \\ Gaetano Assanto \\ Department of Electronic Engineering, Terza University of Rome, 00146 Roma, Italy \\ W. Sohler \\ Angewandte Physik, Universitaet-GH Paderborn, D-33098 Paderborn, Germany
}

(Received 19 March 1998; accepted for publication 27 April 1998)

An all-optically induced spatial shift in the interference between the two lowest-order modes of a lithium niobate channel waveguide was demonstrated based on the power-dependent nonlinear phase shift induced via quadratic cascading. (C) 1998 American Institute of Physics.

[S0003-6951(98)00926-7]

It was pointed out that in the early days of nonlinear optics by Ostrovskii that a nonlinear phase shift can be induced on a fundamental beam when it participates in a nearphase-matched second-harmonic-generation (SHG) process. ${ }^{1}$ It took almost 30 years before this effect, now called cascading, was proposed for all-optical, guided wave, switching devices. $^{2-4}$ One of the drawbacks was that the phase shift required strong coupling to, and significant generation of, a second-harmonic wave which acts like a loss for the fundamental beam, reducing the fundamental beam throughput. However, a variation in the SHG phase-matching condition with propagation distance turned out to be almost ideal for minimizing the loss to the harmonic commensurate with obtaining a large phase shift. ${ }^{5}$ This led to the successful experimental demonstration of both a nonlinear Mach-Zehnder interferometer and a nonlinear directional coupler in birefringently phase-matched $\mathrm{LiNbO}_{3}$ channel waveguides. ${ }^{6}$

A nonlinear mode mixer consists of a single-channel waveguide fabricated to support the two lowest-order guided modes, for example the $\mathrm{TM}_{00}(\omega)$ and $\mathrm{TM}_{01}(\omega)$ modes. Its operational principles are sketched in Fig. 1. The key is that the $\operatorname{TM}_{01}(\omega)$ mode has a two-lobed field distribution with the lobes $\pi$ out of phase with one another. When both the $\mathrm{TM}_{00}(\omega)$ and $\mathrm{TM}_{01}(\omega)$ are launched, and with the $\operatorname{TM}_{00}(\omega)$ wave in phase with one of the lobes of the $\operatorname{TM}_{01}(\omega)$ mode, the total field at the input is localized to one side of the channel because of interference between the two modes. Furthermore, because the two modes propagate with different wave vectors, $\beta_{00}$ and $\beta_{01}$, then the field maximum "switches" periodically back and forth across the waveguide with distance with period $L_{c}=2 \pi /\left[\beta_{00}-\beta_{01}\right]$, see Fig. 1. If, in addition, there is a power-dependent $(P$-dependent $)$ relative phase shift between the modes, $\phi^{N L}(L, P)$, then the total relative phase between the modes is $L\left[\beta_{00}-\beta_{01}\right]+\phi^{N L}(L, P)$. All-optical switching is obtained when $\phi^{N L}\left(L, P_{s}\right)-\phi^{N L}(L, O)=\pi$ so that the field maximum moves from one side of the waveguide to the

${ }^{a}$ Current address: Dept. of Physics, Washington State University, Pullman, WA 99164-2814. other when the power is increased to $P_{s}$. This type of device has been demonstrated before in semiconductors using thirdorder nonlinearities. ${ }^{7}$ Here, we demonstrate this operation in a $\mathrm{LiNbO}_{3}$ channel waveguide using quadratic cascading to generate the additional relative phase shift. ${ }^{8}$

The $15 \mu \mathrm{m}$ wide, titanium in-diffused, $\mathrm{LiNbO}_{3}$ channel waveguide used can support the $\operatorname{TM}_{00}(\omega)$ and $\operatorname{TM}_{01}(\omega)$ modes at the fundamental wavelength $(\lambda=1.32 \mu \mathrm{m})$. Since $\beta_{00} \neq \beta_{01}$, the wave-vector mismatch for SHG is different for each mode at a specific phase-matching temperature. Figure 2 shows the calculated effective indices as a function of temperature for several different fundamental and $\mathrm{SH}$ modes over the temperature range of interest here. Over a $15 \mathrm{~K}$ temperature range there are many possible phase-matching resonances involving the $\mathrm{TM}_{00}(\omega)$ and $\mathrm{TM}_{01}(\omega)$ modes whose strength depends on the respective overlap integrals. At a fixed temperature the wave-vector mismatch is very different for the various SHG processes and different amounts of nonlinear phase shift can be obtained in the fundamental modes for each interaction. This is the origin of the differential power-dependent phase shift between the modes.

To launch each mode separately, the experimental setup shown in Fig. 3 was employed. A $Q$-switched, mode-locked $\mathrm{Nd}$ :YAG laser with a pulse slicer produced $90 \mathrm{ps}$ pulses at a $500 \mathrm{~Hz}$ repetition rate. The waveguides were placed in an oven, which produced a spatial dispersion in the wavevector-matching condition along the waveguide. This led to

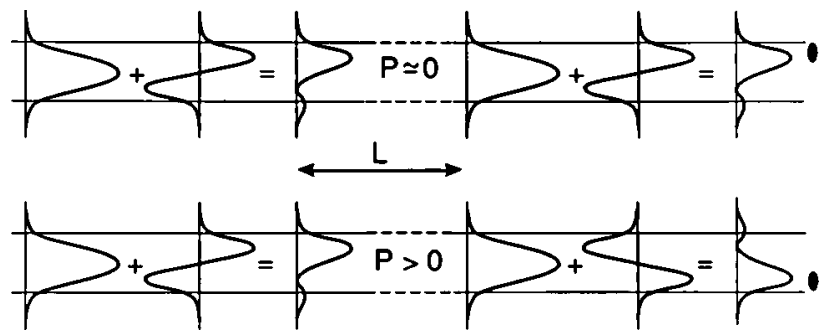

FIG. 1. Principle of a nonlinear mode mixer. When the input power $P$ is increased, the field maximum switches from one side of the waveguide to the other due to the additional power-induced $\pi$ phase shift between the modes. 


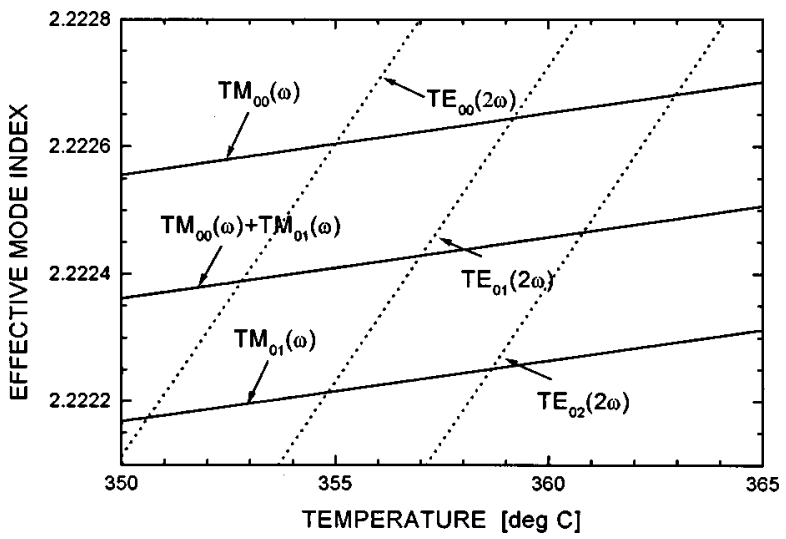

FIG. 2. Calculated effective mode indices for the fundamental and second harmonic as a function of temperature in a $15 \mu \mathrm{m}$ wide waveguide.

large nonlinear phase shifts at fundamental depletions of less than $10 \% .^{5}$ The $\mathrm{TM}_{00}(\omega)$ mode was excited via arm 1 . By adjusting mirror $\mathrm{M} 1$ in arm 2, the higher-order mode was excited by launching an incident beam with a small angle to the waveguide axis. The launching of each mode was verified by imaging the output from the waveguide into a camera and using the half-wave plate before the polarizing beam splitter, the power fraction between the two arms was adjusted carefully to make the output power equal for each mode.

Different SHG tuning curves with launching $\operatorname{TM}_{00}(\omega)$ and $\mathrm{TM}_{01}(\omega)$ separately as well as together were measured to verify the location of the resonance temperatures in the vicinity of the dominant $2 \mathrm{TM}_{00}(\omega) \rightarrow \mathrm{TE}_{00}(2 \omega)$ interaction. Figure 4 shows the SHG tuning curve when both $\mathrm{TM}_{00}$ and $\mathrm{TM}_{01}$ modes were launched. There are two major contributions, the dominant one from $2 \mathrm{TM}_{00}(\omega) \rightarrow \mathrm{TE}_{00}(2 \omega)$ at $336.7^{\circ} \mathrm{C}$ and a secondary one at $337.8^{\circ} \mathrm{C}$ corresponding to the $\mathrm{TM}_{00}(\omega)+\mathrm{TM}_{01}(\omega) \rightarrow \mathrm{TE}_{01}(2 \omega)$ resonance. The resonance from $2 \mathrm{TM}_{01}(\omega) \rightarrow \mathrm{TE}_{02}(2 \omega)$ occurs at $338.8^{\circ} \mathrm{C}$. Other resonances involving the $\mathrm{TM}_{01}(\omega)$ mode only lie at even higher or much lower temperatures. Note that due to inaccuracies in the Sellmeier equations used, ${ }^{9}$ the calculated phase-matching temperatures differ by $\sim 18 \mathrm{~K}$ with respect to the experimental values (see Fig. 2) $336.5^{\circ} \mathrm{C}$. Based on this tuning curve, the experimental temperature was set at $336.5^{\circ} \mathrm{C}$. At this temperature the nonlinear phase shift for any $\operatorname{TM}_{01}(\omega)$ mode interaction is expected to be small compared to that of the $\mathrm{TM}_{00}$ mode, because the resonances for

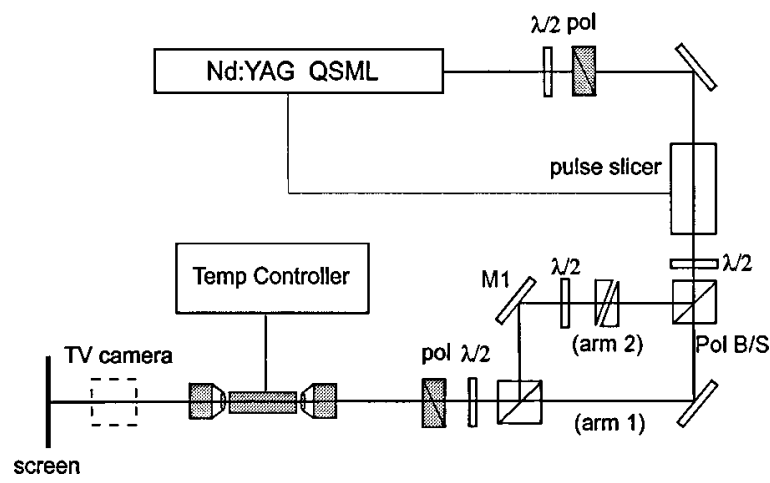

FIG. 3. Experimental setup for all-optical switching by coherent mode mixing.

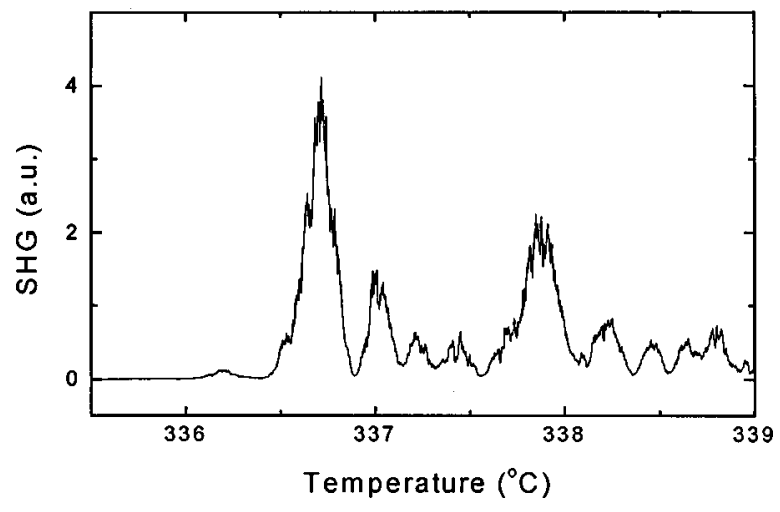

FIG. 4. SHG tuning curve for launching both the $\mathrm{TM}_{00}$ and $\mathrm{TM}_{01}$ fundamental modes.

the $\operatorname{TM}_{01}(\omega)$ are relatively far away and the SHG efficiency is also quite small compared to that for the $\mathrm{TM}_{00}(\omega)$ mode. This feature allows an optimized relative nonlinear phase difference between the modes.

First the relative input phase between the modes was adjusted so that the field maximum at the output could be located on either side of the waveguide. With proper adjustment of the wedge plate in arm 2 (see Fig. 3), the output can exit either the right or left side of the channel as shown in Fig. 5. Different linear interference conditions occur between the modes when the relative phase of the two launched modes changes by $\pi$. Figure 6 compares the normalized output intensity pattern at high ( $340 \mathrm{~W}$ in each mode) and low input powers keeping the input phase of the two modes unchanged. As expected, the location of the output was modified due to the different amounts of nonlinear phase shift accumulated for each mode through cascading. This is in excellent agreement with previous measurements in Ref. 6 and calculations in which the nonlinear phase change in the $\mathrm{TM}_{01}(\omega)$ mode was completely ignored, such that a switching power of around $300 \mathrm{~W}$ (corresponding to an induced $\pi$ phase shift) was estimated.

The output data in Fig. 6 clearly show that the highpower output beam profile is broader than that of the linear output, and asymmetric. This is a direct consequence of the pulsed excitation used in this experiment. Only the peak power portion of the pulse is switched completely over, with the switching efficiency decreasing with decreasing power along the pulse envelope. This results in a long tail to the

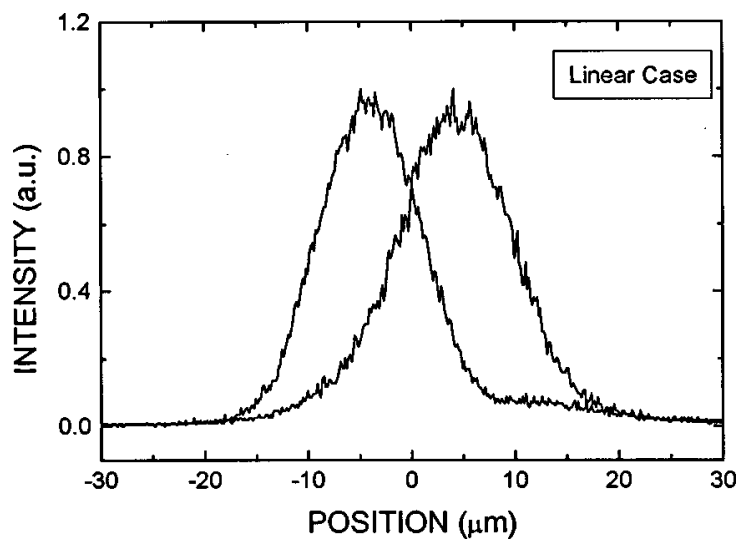

FIG. 5. Output intensity pattern for the low-power case and a phase difference of the modes at the output of 0 and $\pi$, respectively. 


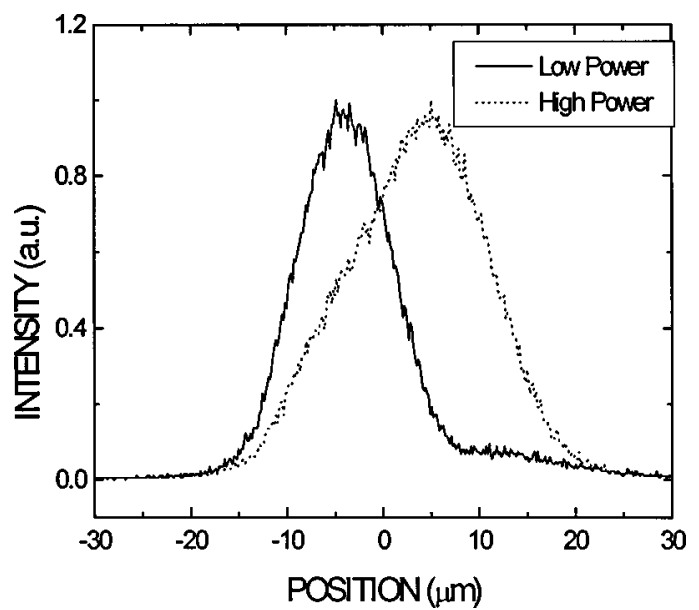

FIG. 6. Normalized output modal interference pattern at $340 \mathrm{~W}$ input power compared to the pattern for low power for the same initial relative phase difference.

output intensity pattern, stretching toward the side of the channel which corresponds to the low-power intensity maximum.

In summary, we have demonstrated spatial switching by the nonlinear mixing of the two lowest-order guided modes in a channel waveguide. The prevailing nonlinearity was cascading, i.e., the nonlinear phase shift accumulated by the fundamental beam near a SHG phase-matching condition. Note that if a $Y$ junction is fabricated at the end of the waveguide to separate the outputs, an all-optical switching device can be built which changes its output port according to input power.

This research was supported by DARPA and ARO.

${ }^{1}$ L. A. Ostrovskii, JETP Lett. 5, 272 (1967).

${ }^{2}$ G. Assanto, G. I. Stegeman, M. Sheik-Bahae, and E. W. Van Stryland, Appl. Phys. Lett. 62, 1323 (1993).

${ }^{3}$ R. Schiek, Opt. Quantum Electron. 26, 415 (1994).

${ }^{4}$ C. N. Ironside, J. S. Aitchison, and J. M. Arnold, IEEE J. Quantum Electron. 29, 2650 (1993).

${ }^{5}$ R. Schiek, M. L. Sundheimer, D. Y. Kim, Y. Baek, G. I. Stegeman, H. Seibert, and W. Sohler, Opt. Lett. 19, 1949 (1994).

${ }^{6}$ Y. Baek, R. Schiek, and G. I. Stegeman, Opt. Lett. 20, 2168 (1995); R. Schiek, Y. Baek, G. Krijnen, G. I. Stegeman, I. Baumann, and W. Sohler, ibid. 21, 940 (1996).

${ }^{7}$ For example, P. LiKamWa, A. Miller, C. B. Park, J. S. Roberts, and P. N. Robson, Appl. Phys. Lett. 57, 1846 (1990).

${ }^{8}$ A. De Rossi, C. Conti, and G. Assanto, Opt. Quantum Electron. 29, 53 (1997).

${ }^{9}$ Y. Baek, Ph.D. thesis, University of Central Florida, 1997. 